\title{
Invited Talk Abstracts
}

\section{General Conference Invited Talks}

\author{
Field Notes: Organizational Models for Responsible AI \\ Susan Etlinger (Altimeter, a Prophet Company, \& Centre for International Governance \\ Innovation, USA)
}

During the past year, we've seen a dramatic increase in public conversation about the ethics of Artificial Intelligence (AI). Concerns about the impact of biased algorithms, lack of transparency, and how AI technologies are being and should be used have consistently garnered headlines in major news outlets around the world. But while news coverage has raised awareness of ethical issues of AI, it generally hasn't addressed the implications for the organizations that buy, build, and implement these technologies.

This talk, based on interviews with leaders from business, academia, and non-governmental organizations, defines issues resulting from the development and deployment of AI, lays out the methodologies being tested and used, and proposes an approach to address them. While this is a highly complex field and no single strategy is appropriate for all industries or companies, this material may be used to better understand the unique implications of AI, begin to socialize them within your business, and build organizational capability to foster both trust and innovation among customers, employees, shareholders, partners, and the general public.

\section{TAKEAWAYS}

Why and how AI changes the relationship between people and organizations The biggest risks - and opportunities - for business in the age of AI Operating principles, recommendations and best practices for designing innovative, engaging and ethical products, services and brand experiences.

\section{SOURCES}

The content is based on a research report entitled "Innovation + Trust: The Foundation of Responsible Artificial Intelligence," which is based on interviews with leaders in academia, industry and the non-profit community.

\section{Deep Learned Models from Medical Images: Successes and Big Challenges Lawrence O. Hall (Co-Director Institute for Artificial Intelligence, Department of Computer Science and Engineering, University of South Florida, USA)}

Medical images may consist of camera-like images (e.g. Dermoscopy), Computed Tomography images (e.g. lung cancer screening), X-ray images (e.g. chest imaging), magnetic resonance images (e.g. brain imaging) and more. This talk will cover how deep learning approaches applied to medical images can be used as an aid to diagnosis and treatment planning. There are medical problems where models learned from images have performance nearly equivalent to the very best medical experts (e.g. dermatology) and others where they can provide useful information that is not typically provided by physicians (e.g. future lung nodule progression). There remain challenges in acquiring good data for training and data that is diverse enough to build a robust classifier. An analysis of pitfalls and potential solutions focused on diagnosing COVID-19 from images will be presented. 
While there's been recent explosion of commercial interest in artificial intelligence (AI), the Department of Defense (DoD) has been steadily exploring AI and its application to national defense for decades. However, recent changes in the international investment in AI, recent technological advances, and the opportunity to leverage the explosion of commercial research efforts has made AI a high priority for both science \& technology and acquisition organizations across the DoD. The problems and constraints posed by national defense applications present challenges for AI that demand technological innovations beyond the current state of the art and beyond what will be achieved by commercial research alone. In many cases, the current challenges encountered in applying AI to national defense will be future challenges for commercial applications. Drawing on almost 30 years of experience working on AI for national defense, Dr. Michael van Lent will discuss the motivations behind the increased emphasis on $\mathrm{AI}$ in the DoD and present examples of the challenges encountered in applying AI to national defense and the research innovations that are solving these challenges. 